\title{
Carnitine, acetylcarnitine and the activity of carnitine acyltransferases in seminal plasma and spermatozoa of men, rams and rats
}

\author{
D. E. Brooks \\ Department of Animal Physiology, The University of Adelaide, Waite Agricultural Research \\ Institute, Glen Osmond, South Australia 5064
}

\begin{abstract}
Summary. The concentration of total carnitine (i.e. carnitine plus acetylcarnitine) was measured in seminal plasma and spermatozoa of men and rams. In ram semen, there was a close correlation between the concentration of spermatozoa and that of total carnitine in the seminal plasma, indicating that the epididymal secretion was the sole source of seminal carnitine. The percentage of total carnitine present as acetylcarnitine was $40 \%$ in seminal plasma and $70-80 \%$ in spermatozoa. The acetylation state of carnitine in seminal plasma was apparently not influenced by the metabolic activity of spermatozoa in ejaculated ram semen as no change was found in the plasma concentration of carnitine or acetylcarnitine up to $45 \mathrm{~min}$ after ejaculation. In spermatozoa, the activity of carnitine acetyltransferase (EC 2.3.1.7) was approximately equivalent to that of carnitine palmitoyltransferase (EC 2.3.1.21); and the activity of these enzymes was similar in ram and human spermatozoa but greater in rat spermatozoa. It is concluded that there is no correlation between the content of either total carnitine or the carnitine acyltransferases and the respiratory capacity of spermatozoa.
\end{abstract}

\section{Introduction}

High levels of carnitine (up to $60 \mathrm{~mm}$ ) are found in the excurrent duct system of the male rat (Marquis \& Fritz, 1965; Brooks, Hamilton \& Mallek, 1974) and several other species (Casillas, 1972). Particularly in the rat, but also in other species, most of the carnitine occurs in the luminal fluid of the epididymis (Marquis \& Fritz, 1965; Brooks et al., 1974; Hinton, Snoswell \& Setchell, 1979). Nevertheless the concentration of carnitine plus acetylcarnitine within spermatozoa is substantial, being of the order of 40-100 mm (Hutson, Van Dop \& Lardy, 1977; Brooks, 1979).

The acetylation state of carnitine within spermatozoa can be markedly influenced by a variety of exogenous substrates (Casillas \& Erickson, 1975; Milkowski, Babcock \& Lardy, 1976; Van Dop, Hutson \& Lardy, 1977). Furthermore, acetylcarnitine can act as a ready source of oxidizable acetyl units for spermatozoa (Storey \& Keyhani, 1974; Hutson et al., 1977) in addition to acting as a buffer for acetyl CoA levels (Casillas \& Erickson, 1975). In the absence of a glycolysable substrate, spermatozoa can also oxidize fatty acids liberated from endogenous choline plasmalogen (Hartree \& Mann, 1959, 1961), presumably involving carnitine as a cofactor. At least two carnitine acyltransferases are involved in the formation of fatty-acyl carnitine. Carnitine acetyltransferase (EC 2.3.1.7) catalyses the formation and utilization of acetylcarnitine and esters of other short-chain fatty acids, whereas carnitine palmitoyltransferase (EC 2.3.1.21) is required for the formation of long-chain fatty-acyl carnitine and thereby facilitates the transport of long-chain fatty-acids into the mitochondrion to the site of $\beta$-oxidation. 
Despite the apparent importance of carnitine in sperm metabolism, measurements of carnitine and acetylcarnitine in seminal plasma have been made only in the monkey and man (Frenkel, Peterson, Davis \& Freund, 1974; Casillas \& Erickson, 1975: Lewin, Beer \& Lunenfeld, 1976; Kohengkul, Tanphaichitr, Muangmun \& Tanphaichitr, 1977; Wetterauer \& Heite, 1978; Suter \& Holland, 1979). Some estimates of the activities of the carnitine acyltransferases have been made in rat and human spermatozoa (Marquis \& Fritz, 1965; Geer, Kelley, Pohlman \& Yemm, 1975; Brooks, 1978; Bøhmer, Johansen \& Kjekshus, 1978), but due to differences in the assay procedures and the manner of expressing the results, it is difficult to make direct comparisons between these reports. The present work was undertaken to extend the observations which have been made on men and rats, to explore the role of carnitine and acetylcarnitine in seminal plasma, and to examine whether the respiratory activity of spermatozoa is related to their content of the carnitine acyltransferases or of carnitine plus acetylcarnitine. The semen of men and rams was chosen because spermatozoa from these two sources differ markedly in their respiratory activity and in their concentration per unit volume of semen.

\section{Materials and Methods}

Enzymes and coenzymes were obtained from Boehringer Mannheim Australia Pty. Ltd, Melbourne, Australia. L-Carnitine and L-acetylcarnitine were donated by $\operatorname{Dr}$ A. M. Snoswell, Department of Agricultural Biochemistry, University of Adelaide, South Australia. Ficoll 400 was supplied by Pharmacia (South Seas) Pty. Ltd, North Ryde, New South Wales, Australia. Other chemicals were of analytical reagent grade.

Semen was collected from 12 Merino rams by electrical stimulation (Blackshaw, 1954) and from 3 men ( 2 of proven fertility) by masturbation. Human semen was allowed to liquefy ( $30-45$ min at room temperature) before further treatment. Rat spermatozoa were recovered from the epididymis as described by Brooks (1978).

Seminal plasma and spermatozoa were separated by centrifugation of semen for $2.5 \mathrm{~min}$ at $10000 \mathrm{~g}$ in a Microfuge (Beckman Instruments Ltd, Palo Alto, California, U.S.A.). Two volumes of $10 \%(\mathrm{w} / \mathrm{v})$ trichloroacetic acid were added to the seminal plasma to precipitate proteins.

To obtain spermatozoa for study, semen was diluted with $1-3$ volumes of $0.15 \mathrm{M}-\mathrm{NaCl}$ which was then layered over a Ficoll medium and centrifuged as described by Harrison (1976). For enzyme assay the supernatant was rapidly aspirated and the tube containing the sedimented spermatozoa was frozen until required. For the determination of intracellular carnitine and acetylcarnitine, the supernatant was largely removed but the spermatozoa were resuspended in the Ficoll medium $(0.5 \mathrm{ml}$ total volume), sometimes aided by gentle shaking of the tube. About 10 min after resuspension, $1 \mathrm{ml} \mathrm{10 \%} \mathrm{trichloroacetic} \mathrm{acid} \mathrm{was} \mathrm{added} \mathrm{and} \mathrm{the} \mathrm{precipitated} \mathrm{material}$ was removed by centrifugation at $1500 \mathrm{~g}$ and $2{ }^{\circ} \mathrm{C}$ for $10 \mathrm{~min}$.

Excess trichloroacetic acid was removed from deproteinized extracts by 6 extractions with an equal volume of water-saturated ether. Excess ether was then removed from the samples under a stream of $\mathrm{N}_{2}$.

Carnitine was measured as described by Marquis \& Fritz (1964) and acetylcarnitine by the procedure of Pearson \& Tubbs (1964) as modified by Pearson (1965). Carnitine acetyltransferase and carnitine palmitoyltransferase were extracted and assayed as described by Brooks (1978) except that their activity was determined in the total homogenate rather than in the $600 \mathrm{~g}$ supernatant. Enzyme activity was expressed as units $/ 10^{\circ}$ spermatozoa, where a unit is defined as $1 \mu \mathrm{mol}$ substrate converted $/ \mathrm{min}$ at $25^{\circ} \mathrm{C}$.

Sperm numbers were estimated after diluting small aliquots of semen, or washed spermatozoa, in $0.15 \mathrm{M}-\mathrm{NaCl}$ containing $0.2 \%(\mathrm{w} / \mathrm{v})$ formaldehyde. Human and rat spermatozoa were counted in a haemocytometer whereas the number of ram spermatozoa was 
estimated by measuring the absorption of the diluted suspension at $650 \mathrm{~nm}$ in a spectrophotometer and then using an equation derived from a previous calibration against haemocytometer counts (Bishop, Campbell, Hancock \& Walton, 1954).

\section{Results}

The level of total carnitine (i.e. carnitine + acetylcarnitine) was about 10 times greater in the seminal plasma of rams compared with that of men (Table 1) but the proportion present as acetylcarnitine was similar. For ram semen, the level of total carnitine in the seminal plasma was highly correlated with the sperm density (Text-fig. 1). The amounts of carnitine and acetylcarnitine were similar in human and ram spermatozoa (Table 1). The acetylation state of carnitine in spermatozoa was higher than in seminal plasma. However, as the spermatozoa were separated from the seminal plasma into a different medium, the two results are not directly comparable.

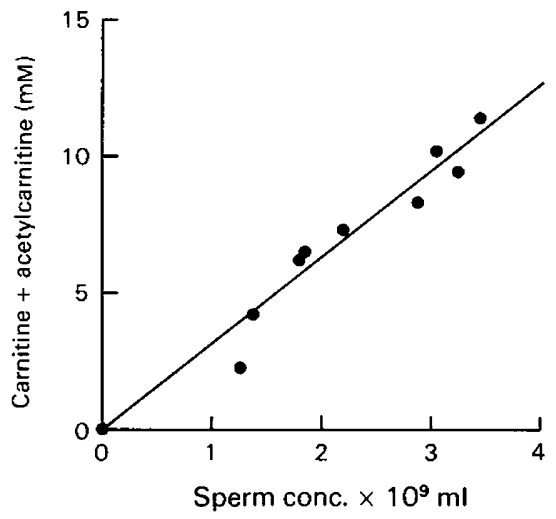

Text-fig. 1. Relationship between sperm concentration and total carnitine (carnitine + acetylcarnitine) in ram semen. Each point represents a determination made on the ejaculate of a different ram. The point at zero sperm concentration represents 2 determinations which were made on semen collected from 2 vasectomized rams; these values were omitted from the calculation of the mean value given in Table 1. The straight line represents a fitted linear regression with correlation coefficient of 0.96 .

In order to establish whether the acetylation state of carnitine in seminal plasma is influenced by the metabolic activity of the spermatozoa after ejaculation, carnitine and acetylcarnitine were measured in ram seminal plasma after separation from the spermatozoa at various times after ejaculation. No change was found either in the acetylation state of carnitine or in the concentration of carnitine plus acetylcarnitine between 1.5 and 45 min after ejaculation (Textfig. 2). It was assumed that the spermatozoa contained in this sample of semen were metabolically active as they demonstrated excellent wave motion when observed under lowpower magnification.

In the spermatozoa of all species examined, the activity of carnitine acetyltransferase was similar to that of carnitine palmitoyltransferase (Table 1). There was, however, a difference in the degree of binding of these eznymes within the spermatozoa. Despite the fact that these spermatozoa had been frozen and thawed, treated with detergent and then sonicated, only $20 \%$ of the carnitine palmitoyltransferase was recovered in the supernatant $(600 \mathrm{~g}, 10 \mathrm{~min})$ from ram spermatozoa and $50 \%$ from human spermatozoa, whereas $75 \%$ or more of the carnitine 
Table 1. Carnitine, acetylcarnitine, carnitine acetyltransferase and carnitine palmitoyltransferase in seminal plasma and spermatozoa of men, rams and rats

\begin{tabular}{|c|c|c|c|c|c|}
\hline \multirow[b]{2}{*}{$\begin{array}{c}\text { Parameter } \\
\text { measured }\end{array}$} & \multicolumn{2}{|c|}{ Seminal plasma } & \multicolumn{3}{|c|}{ Spermatozoa } \\
\hline & $\underset{(6)}{\operatorname{Man}}$ & $\begin{array}{c}\operatorname{Ram} \\
(9)\end{array}$ & $\underset{(3)}{\operatorname{Man}}$ & $\underset{(7)}{\operatorname{Ram}}$ & $\begin{array}{l}\text { Rat } \\
\text { (4) }\end{array}$ \\
\hline Sperm density $\left(\times 10^{9} / \mathrm{ml}\right)$ & $0.14 \pm 0.01$ & $2 \cdot 34 \pm 0.28$ & $0.14 \pm 0.02$ & $2.63 \pm 0.26$ & - \\
\hline Carnitine* & $0.31+0.06$ & $4.5 \pm 0.7$ & $0.25 \pm 0.08$ & $0.65+0.22$ & _- \\
\hline Acetylcarnitine* & $0.18 \pm 0.03$ & $2.8 \pm 0.4$ & $1.23 \pm 0.50$ & $1.09 \pm 0.16$ & - \\
\hline Carnitine + acetylcarnitine & $0.49 \pm 0.03$ & $7 \cdot 3 \pm 1 \cdot 0$ & $1.48 \pm 0.57$ & $1.73 \pm 0.34$ & - \\
\hline$\%$ acetylation of carnitine & $39 \pm 8$ & $40 \pm 4$ & $82 \pm 2$ & $67 \pm 5$ & - \\
\hline Carnitine acetyltransferase $†$ & 二 & - & $0.93 \pm 0.16$ & $1.28 \pm 0.14$ & $7.58 \pm 0.71$ \\
\hline \multirow{2}{*}{$\begin{array}{l}\text { Carnitine palmitoyl- } \\
\text { transferase }\end{array}$} & & & & & \\
\hline & - & - & $0.84 \pm 0.05$ & $1.90 \pm 0.16$ & $5 \cdot 90 \pm 0.5$ \\
\hline
\end{tabular}

Values are given as the mean \pm s.e.m. and the number of replicates is given by the figure in parentheses. Each determination was made on the ejaculate of a different animal, except for human seminal plasma where 2 ejaculates were obtained from each volunteer.

${ }^{*}$ Measured in mM in seminal plasma and $\mu \mathrm{mol} / 10^{9}$ spermatozoa.

$\dagger$ Measured in units (see text) $/ 10^{9}$ spermatozoa.

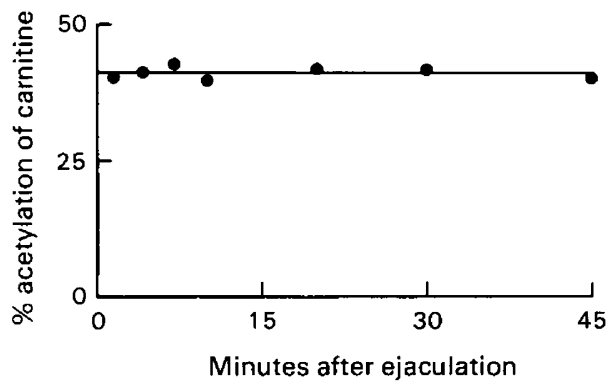

Text-fig. 2. The percentage acetylation of carnitine in ram seminal plasma at various times after ejaculation. Aliquots $(50 \mu \mathrm{l})$ of semen were centrifuged in glass capillary tubes for $1 \mathrm{~min}$ at $10000 \mathrm{~g}$ to sediment the spermatozoa. The seminal plasma was transferred to a second capillary and immersed in a boiling water bath for $1 \mathrm{~min}$ to denature proteins. Aliquots were subsequently taken for the determination of carnitine and acetylcarnitine.

acetyltransferase was released by this treatment. Due to the incomplete release of activity, measurements of the carnitine acyltransferases were made using the complete homogenate.

\section{Discussion}

The amount of carnitine plus acetylcarnitine found in human and ram spermatozoa in this study is similar to that found in other species (Casillas, 1972, 1973; Brooks et al., 1974; Casillas \& Erickson, 1975; Milkowski et al., 1976; Van Dop et al., 1977). Despite the similarity of the content of carnitine plus acetylcarnitine in human and ram spermatozoa, the concentration of these two substances in seminal plasma is about 10 times greater for the ram than man (Table 1).

Since ejaculated spermatozoa are derived only from the ductus deferens and epididymis, the direct correlation between the concentration of carnitine plus acetylcarnitine in seminal plasma and the concentration of spermatozoa in ram ejaculates (Text-fig. 1) suggests that the ductus 
deferens and epididymis are also the sole source of seminal carnitine. This was confirmed by the lack of carnitine in semen collected from vasectomized rams. Similarly, in the rat the epididymis is probably the only source of carnitine for the ejaculate because the levels of carnitine in the seminal vesicle, coagulating gland and prostate are low (Brooks et al., 1974; Brooks \& McIntosh, 1975) and what little carnitine they contain may be associated with the tissues themselves rather than with their secretions. In men, however, there is little correlation between sperm numbers and the concentration of carnitine in seminal plasma (Wetterauer \& Heite, 1978; Suter \& Holland, 1979), and the concentration of carnitine in the seminal plasma of vasectomized men is only half that of normal males (Frenkel et al., 1974). This indicates a substantial contribution of carnitine to the seminal plasma of men by accessory glands other than the epididymis.

As the concentration of carnitine in the seminal plasma of a sample of ram semen remained constant for at least 45 min after ejaculation, it can be assumed that the plasma carnitine did not arise by leakage from the spermatozoa. Moreover, although the ejaculate contained highly active spermatozoa, there was no change in the concentration of acetylcarnitine in the seminal plasma. This indicates that the spermatozoa were not using this extracellular acetylcarnitine as an energy substrate. The acetylation state of carnitine $(40 \%)$ in ram seminal plasma can therefore be taken as equivalent to that which exists within the epididymis, unless major changes had occurred within the short period $(1.5 \mathrm{~min})$ after the mixing of the epididymal contents with the secretions of the other accessory glands at ejaculation but before the first sampling. By comparison, the acetylation state of carnitine within the luminal fluid of the rat epididymis has been estimated to be $10 \%$ (Brooks et al., 1974).

In contrast to ejaculated spermatozoa, epididymal spermatozoa are considerably more permeable to carnitine, especially those spermatozoa recovered from more proximal regions of the duct (Casillas, 1973). Within these regions of the epididymis an equilibrium of carnitine and acetylcarnitine could be expected to form between the spermatozoa and the surrounding fluid. A low acetylation state of carnitine within the epididymal fluid would then buffer the acetyl CoA of spermatozoa at a low level (Casillas \& Erickson, 1975) and this might be a factor contributing towards the restricted metabolic activity of spermatozoa which is presumed to exist within the epididymis.

Carnitine acetyltransferase is particularly active in spermatozoa compared with other tissues (Marquis \& Fritz, 1965; Brooks, 1978) and spermatozoa can readily use intracellular acetylcarnitine as a source of energy (Milkowski et al., 1976). Bøhmer et al. (1978) detected some carnitine acetyltransferase in human seminal plasma and this activity represented about $1 \%$ of the concentration present in spermatozoa. Bøhmer et al. consider it likely that the activity in seminal plasma arose by leakage from dead spermatozoa. In the present study, carnitine palmitoyltransferase has been shown to have an activity in spermatozoa equivalent to that of carnitine acetyltransferase. However, the role of carnitine palmitoyltransferase in the metabolism of spermatozoa remains to be fully clarified since differing effects of carnitine on long-chain fatty-acid oxidation in spermatozoa have been reported. For instance, the rate of palmitate oxidation is reduced by the addition of carnitine to ejaculated bovine spermatozoa (Hamilton \& Olson, 1976) but is enhanced by a corresponding addition to epididymal spermatozoa (Casillas, 1972). Furthermore, the mitochondria of rabbit epididymal spermatozoa appear to lack the outer carnitine palmitoyltransferase, since they can oxidize palmitoylcarnitine but not palmitoyl CoA in the presence of carnitine (Storey \& Keyhani, 1974).

It is apparent that the respiratory activity of spermatozoa is related neither to their content of carnitine acyltransferases, nor to their content of carnitine plus acetylcarnitine. For example, there is no large difference between the activity of the carnitine acyltransferases in ram and human spermatozoa nor in their content of carnitine plus acetylcarnitine, although the respiratory activity of ram spermatozoa (about $50 \mu \mathrm{l} \mathrm{O} / 10^{8}$ spermatozoa per $\mathrm{h}$ at $37^{\circ} \mathrm{C}$ ) is approximately 10 times greater than that of human spermatozoa (e.g. Scott, White \& Annison, 1962; Murdoch 
\& White, 1968; Peterson \& Freund, 1968; Eliasson, 1970). Moreover, the activity of the carnitine acyltransferases is considerably greater in the spermatozoa of rats than in those of men and rams, yet the respiratory rate of rat spermatozoa $\left(7 \mu \mathrm{O} \mathrm{O}_{2} / 10^{8}\right.$ spermatozoa per $\mathrm{h}$ at $25^{\circ} \mathrm{C}$; Brooks, 1978) is intermediate between that of human and ram spermatozoa.

This work was supported by the Australian Research Grants Committee. A Microfuge was purchased in part by a Grant-in-Aid of Research from Sigma Xi, the Scientific Research Society of North America.

\section{References}

Bishop, M.W.H., Campbell, R.C., Hancock, J.L. \& Walton, A. (1954) Semen characteristics and fertility in the bull. J. agric. Sci., Camb. 44, 227-248.

Blackshaw, A.W. (1954) A bipolar rectal electrode for the electrical production of ejaculation in sheep. Aust. vet. J. 30, 249-250.

Bøhmer, T., Johansen, L. \& Kjekshus, E. (1978) Carnitine-acetyl-transferase in human spermatozoa and seminal plasma determined by a sensitive radioisotope method. Int. J. Andrology 1, 262-269.

Brooks, D.E. (1978) Activity and androgenic control of enzymes associated with the tricarboxylic acid cycle, lipid oxidation and mitochondrial shuttles in the epididymis and epididymal spermatozoa of the rat. Biochem. J. 174, 741-752.

Brooks, D.E. (1979) Biochemical environment of sperm maturation. In The Spermatozoon. Eds D. W. Fawcett \& J. M. Bedford. Urban \& Schwarzenburg, Baltimore.

Brooks, D.E. \& McIntosh, J.E.A. (1975) Turnover of carnitine by rat tissues. Biochem. J. 148, 439-445.

Brooks, D.E., Hamilton, D.W. \& Mallek, A.H. (1974) Carnitine and glycerylphosphorylcholine in the reproductive tract of the male rat. $J$. Reprod. Fert. 36, 141-160.

Casillas, E.R. (1972) The distribution of carnitine in male reproductive tissues and its effect on palmitate oxidation by spermatozoal particles. Biochim. Biophys. Acta 280, 545-551.

Casillas, E.R. (1973) Accumulation of carnitine by bovine spermatozoa during maturation in the epididymis. J. biol. Chem. 248, 8227-8232.

Casillas, E.R. \& Erickson, B.J. (1975) The role of carnitine in spermatozoan metabolism: substrate induced elevations in the acetylation state of carnitine and coenzyme $\mathbf{A}$ in bovine and monkey spermatozoa. Biol. Reprod. 12, 275-283.

Eliasson, R. (1970) Oxygen consumption of human semen. Biol. Reprod. 3, 369-374.

Frenkel, G., Peterson, R.N., Davis, J.E. \& Freund, M. (1974) Glycerylphosphorylcholine and carnitine in normal human semen and in postvasectomy semen: differences in concentrations. Fert. Steril. 25, 84-87.

Geer, B.W., Kelley, K.R., Pohlman, T.H. \& Yemm, S.J. (1975) A comparison of rat and Drosophila spermatozoan metabolisms. Comp. Biochem. Physiol. 50B, 41-50.

Hamilton, D.W. \& Olson, G.E. (1976) Effects of carnitine on oxygen uptake and utilization of [U-
${ }^{14} \mathrm{C}$ lpalmitate by ejaculated bull spermatozoa. $J$. Reprod. Fert. 46, 195-202.

Harrison, R.A.P. (1976) A highly efficient method for washing mammalian spermatozoa. J. Reprod. Fert. 48, 347-353.

Hartree, E.F. \& Mann, T. (1959) Plasmalogen in ram semen, and its role in sperm metabolism. Biochem. $J$. 71, 423-434.

Hartree, E.F. \& Mann, T. (1961) Phospholipids in ram semen: metabolism of plasmalogen and fatty acids. Biochem. J. 80, 464-476.

Hinton, B.T., Snoswell, A.M. \& Setchell, B.P. (1979) The concentration of carnitine in the luminal fluid of the testis and epididymis of the rat and some other mammals. J. Reprod. Fert. 56, 105-111.

Hutson, S.M., Van Dop, C. \& Lardy, H.A. (1977) Mitochondrial metabolism of pyruvate in bovine spermatozoa. J. biol. Chem. 252, 1309-1315.

Kohengkul, S., Tanphaichitr, V., Muangmun, V. \& Tanphaichitr, N. (1977) Levels of L-carnitine and L$\mathrm{O}$-acetylcarnitine in normal and infertile human semen: a lower level of L-O-acetylcarnitine in infertile semen. Fert. Steril. 28, 1333-1336.

Lewin, L.M., Beer, R. \& Lunenfeld, B. (1976) Epididymis and seminal vesicle as sources of carnitine in human seminal fluid: the clinical significance of carnitine concentration in human seminal fluid. Fert. Steril. 27, 9-13.

Marquis, N.R. \& Fritz, I.B. (1964) Enzymological determination of free carnitine concentrations in rat tissues. J. Lipid Res. 5, 184-187.

Marquis, N.R. \& Fritz, I.B. (1965) Effects of testosterone on the distribution of carnitine, acetylcarnitine, and carnitine acetyltransferase in tissues of the reproductive system of the male rat. J. biol. Chem. 240, 2197-2200.

Milkowski, A.L., Babcock, D.F. \& Lardy, H.A. (1976) Activation of bovine epididymal sperm respiration by caffeine. Its transient nature and relationship to the utilisation of acetyl carnitine. Archs Biochem. Biophys. 176, 250-256.

Murdoch, R.N. \& White, I.G. (1968) Studies on the metabolism of human spermatozoa. J. Reprod. Fert. 16, 351-361.

Pearson, D.J. (1965) A source of error in the assay of acetyl-coenzyme A. Biochem. J. 95, 23C-24C.

Pearson, D.J. \& Tubbs, P.K. (1964) Acetyl-carnitine in heart and liver. Nature, Lond. 202, 91.

Peterson, R.N. \& Freund, M. (1968) An evaluation of 
the respiratory capacity of human spermatozoa. $J$. Reprod. Fert. 17, 357-372.

Scott, T.W., White, I.G. \& Annison, E.F. (1962) Glucose and acetate metabolism by ram, bull, dog and fowl spermatozoa. Biochem. J. 83, 398-404.

Storey, B.T. \& Keyhani, E. (1974) Energy metabolism of spermatozoa. II. Comparison of pyruvate and fatty acid oxidation by mitochondria of rabbit epididymal spermatozoa. Fert. Steril. 25, 857-864.

Suter, D.A.I. \& Holland, M.K. (1979) The con- centrations of free L-carnitine and L-Oacetylcarnitine in spermatozoa and seminal plasma of normal, fresh and frozen human semen. Fert. Steril. 31, $541-544$.

Van Dop, C., Hutson, S.M. \& Lardy, H.A. (1977) Pyruvate metabolism in bovine epididymal spermatozoa. J. biol. Chem. 252, 1303-1308.

Wetterauer, U. \& Heite, H.-J. (1978) Carnitine in seminal fluid as parameter for the epididymal function. Andrologia 10, 203-210.

Received 21 December 1978 